\title{
OPEN Pyk2 in dorsal hippocampus plays a selective role in spatial memory and synaptic plasticity
}

\author{
Vincenzo Mastrolia ${ }^{1,2,3,4,8}$, Omar al Massadi ${ }^{1,2,3,5,6,8}$, Benoit de Pins ${ }^{1,2,3,7}$ \& \\ Jean-Antoine Girault ${ }^{1,2,3 凶}$
}

Pyk2 is a $\mathrm{Ca}^{2+}$-activated non-receptor tyrosine kinase enriched in the forebrain, especially in pyramidal neurons of the hippocampus. Previous reports suggested its role in hippocampal synaptic plasticity and spatial memory but with contradictory findings possibly due to experimental conditions. Here we address this issue and show that novel object location, a simple test of spatial memory induced by a single training session, is altered in Pyk2 KO mice and that re-expression of Pyk2 in the dorsal hippocampus corrects this deficit. Bilateral targeted deletion of Pyk2 in dorsal hippocampus CA1 region also alters novel object location. Long term potentiation (LTP) in CA1 is impaired in Pyk2 KO mice using a high frequency stimulation induction protocol but not with a theta burst protocol, explaining differences between previous reports. The same selective LTP alteration is observed in mice with Pyk2 deletion in dorsal hippocampus CA1 region. Thus, our results establish the role of Pyk2 in specific aspects of spatial memory and synaptic plasticity and show the dependence of the phenotype on the type of experiments used to reveal it. In combination with other studies, we provide evidence for a selective role of non-receptor tyrosine kinases in specific aspects of hippocampal neurons synaptic plasticity.

Synaptic function and plasticity are regulated by multiple signaling pathways including numerous protein kinases, among which evidence supports a role for non-receptor tyrosine kinases of the Src family (SFKs) and protein tyrosine kinase $2 \mathrm{~b}$ (Ptk2b) commonly known as Pyk2. Pyk2 is a non-receptor tyrosine kinase closely related to focal adhesion kinase (FAK, Ptk2) but in contrast to FAK, Pyk2 is activated by increased cytoplasmic free $\mathrm{Ca}^{2+1}$. The mechanism of action of $\mathrm{Ca}^{2+}$ may include facilitation of Pyk2 dimerization ${ }^{2,3}$. Depending on cell types, Pyk 2 activation requires the activity of $\mathrm{PKC}^{4}, \mathrm{Ca}^{2+} /$ calmodulin-dependent protein kinases ${ }^{5}$ or calcineurin ${ }^{6}$, but whether these enzymes act directly or indirectly is not known. Pyk2 is highly expressed in the forebrain, especially in hippocampus ${ }^{7}$ where it is activated by neuronal activity $2,8-10$.

Pyk2 and SFKs are functionally linked because activated Pyk2 autophosphorylates at Thr402, and recruits and activates SFKs (review in ${ }^{11}$ ). SFKs potentiate the activity of Pyk2 by phosphorylating its activation loop ${ }^{12}$. SFKs and presumably Pyk2 itself can phosphorylate and modulate NMDA receptors (NMDAR, review in ${ }^{13}$ ). Pyk2 can also interact with many protein partners including PSD-95, which binds Pyk2 proline-rich motif through its Src-homology domain 3 (SH3) ${ }^{14}$. This may anchor Pyk2 to NMDAR with which PSD-95 interacts through its PDZ domain (review in ${ }^{15}$ ). Interestingly $\mathrm{Ca}^{2+}$ can induce the dimerization of PSD-95 and trigger the autophosphorylation of Pyk2 $2^{2}$. Glutamate and NMDA cause post-synaptic clustering of Pyk2, which is prevented by overexpression of PSD-95 SH3 domain ${ }^{2}$. The upregulation of Grin2A (NMDAR subunit 2A, a.k.a. NR2A) induced by SFKs involves PSD-95 phosphorylation and its interaction with Pyk2 ${ }^{16}$.

Several reports provided evidence for a role of Pyk2 in synaptic plasticity, a process in which tyrosine phosphorylation has been known for many years to be implicated ${ }^{17}$. Pyk2 mediates the upregulation of NMDAR currents induced by metabotropic glutamate receptor 1 (Grm1, a.k.a. mGluR1) in cortical neurons in culture ${ }^{18}$.

${ }^{1}$ Inserm UMR-S 1270, 75005 Paris, France. ${ }^{2}$ Sorbonne Université, Faculty of Science and Engineering, 75005 Paris, France. ${ }^{3}$ Institut du Fer à Moulin, 17, rue du Fer à Moulin, 75005 Paris, France. ${ }^{4}$ Present address: Centre for Developmental Neurobiology, MRC Centre for Neurodevelopmental Disorders, New Hunt's House, King's College London, Guy's Campus, London SE1 1UL, UK. ${ }^{5}$ Present address: Instituto de Investigación Sanitaria de Santiago de Compostela, Complexo Hospitalario Universitario de Santiago (CHUS/SERGAS), Travesía da Choupana s/n, 15706 Santiago de Compostela, Spain. ${ }^{6}$ Present address: CIBER Fisiopatologia de la Obesidad y Nutrición (CIBERobn), Madrid, Spain. ${ }^{7}$ Present address: Department of Plant and Environmental Sciences, Weizmann Institute of Science, Rehovot, Israel. ${ }^{8}$ These authors contributed equally: Vincenzo Mastrolia and Omar al Massadi. ${ }^{凶}$ email: jean-antoine.girault@inserm.fr 
In cultured hippocampal neurons brain-derived neurotrophic factor (BDNF) increases NMDAR currents by enhancing local translation of Pyk $2^{19}$. Pyk2 potentiates NMDAR currents in acutely dissociated hippocampal CA1 pyramidal neurons, whereas overexpression of kinase-dead Pyk2 impairs long term potentiation (LTP) in this preparation ${ }^{10}$. Interfering with Pyk2 interaction with PSD-95 also prevents LTP induction ${ }^{2}$. In contrast, in cultured hippocampal slices, Pyk2 knock-down blocks long term depression (LTD) but not LTP induction ${ }^{20}$. These results support a role of Pyk2 in synaptic plasticity but the differences suggest that this role may be dependent on experimental conditions. Recent studies investigated synaptic plasticity in Pyk2 KO mice with diverging conclusions. We reported that in the absence of Pyk2 LTP is altered at Schaffer collaterals-CA1 pyramidal cells synapses $^{21}$, whereas another group using a different Pyk2 KO mouse line found normal LTP but impaired LTD at these synapses ${ }^{22}$. It is important to underline that in these various studies, the protocols used for studying synaptic plasticity were not the same (see "Results" and "Discussion"). In addition, the two studies in Pyk2 KO mice reported different results for hippocampal-dependent memory with impairment of novel object location ${ }^{21}$, but no alteration in the Morris water maze ${ }^{22}$.

In order to clarify the functional role of Pyk 2 and the differences in findings between various reports we examined novel object location (NOL), a simple and sensitive spatial memory test ${ }^{23}$, in mice with a constitutive Pyk2 KO or a selective Pyk2 deletion in the dorsal hippocampus. We also re-examined synaptic plasticity at CA1 synapses and compared in the same mouse line the protocols previously used by different groups in different lines. We show that mice with a constitutive Pyk2 deletion display a NOL deficit that is rescued by Pyk 2 reexpression and mimicked by targeted deletion in the dorsal hippocampus. We also show that the LTP alteration in slices from mice with a Pyk2 $\mathrm{KO}$ or its targeted deletion in CA1 neurons depends on the protocol used for inducing potentiation. Our observations indicate that Pyk2-deficient mice display a specific memory alteration and clarify the role of Pyk2 in synaptic plasticity showing that it is partial and dependent on the experimental protocol used for its induction.

\section{Results}

Spatial memory is altered in Pyk2 knockout mice. We first used a constitutive Pyk2 KO (Ptk2 $\left.2 b^{-1-}\right)$ mouse line and tested its behavioral phenotype using the novel object location test (NOL, Fig. 1A), a task that assesses hippocampus-dependent spatial memory by testing the mouse ability to recognize the repositioning of a familiar object ${ }^{24,25}$. We chose this test, which involves a single brief session of training $(10 \mathrm{~min})$ and is sensitive to dorsal hippocampus alterations ${ }^{23}$, because it was previously reported to be altered in Pyk2 KO mice ${ }^{21}$. In contrast, other commonly used tests, such as novel object recognition (NOR) and Morris water maze, which involve several brain regions or repeated training, respectively ${ }^{23}$ do not appear to be altered in Pyk2 KO mice ${ }^{22,26}$. In the NOL paradigm, as expected, $24 \mathrm{~h}$ after the first presentation, wild type animals spent more time exploring the object moved to the new location (NL) than the other object that remained at the old location (OL). In contrast, after $24 \mathrm{~h}$ Pyk2 KO animals did not show any preference for the objects at either location (Fig. 1B). The mean difference in time spent at NL between WT and KO was $-10.9(95.0 \% \mathrm{CI}-17.3,-4.47)$ with a P value of the two-sided permutation t-test of 0.004 (Supplementary Table 1). These results confirmed the existence of a spatial memory deficit in Pyk2 KO mice as evaluated by the NOL test.

Re-expression of Pyk2 in dorsal hippocampus rescues performance of Pyk2 KO mice in the NOL test. We then thought to determine whether the deficit in the NOL test observed in Pyk2 KO mice could be reversed by re-expression of Pyk 2 in the dorsal hippocampus. To our knowledge, the reversibility of this deficit has not been investigated before. Male 2-4 month-old Pyk2 KO mice received in the dorsal hippocampus a bilateral double stereotactic injection of AAV-GFP/Pyk2 or of AAV-GFP as a control. Three weeks later they were trained for NOL. Twenty-four hours after training, the Pyk2 KO mice injected with AAV-GFP spent the same time exploring the object at the novel and old locations (Fig. 2A), as in the case of non-injected KO mice (see Fig. 1B). In contrast the Pyk2 KO mice injected with AAV-GFP/Pyk2 spent more time exploring the object placed at a novel location than expected by chance (Fig. 2A). The difference in mean time spent at NL between AAV-GFP and AAV-GFP/Pyk2 was $13.8 \%(95.0 \%$ CI 3.2, 24.8) with a P value of the two-sided permutation t-test of 0.024 (Supplementary Table 1). This result shows that the behavioral alteration observed in Pyk2 KO mice is reversible upon re-expression of Pyk2 in the dorsal hippocampus, ruling out permanent developmental alteration or indirect effects. Because the AAV1 serotype used ${ }^{27}$ preferentially targets neurons and the CaMkinase $2 \alpha$ (Camk2a gene) promoter is particularly active in pyramidal neurons ${ }^{28}$, these results suggest that the consequences of the absence Pyk 2 on NOL are linked to its deficit in dorsal hippocampus pyramidal neurons.

Targeted deletion of Pyk2 in dorsal hippocampus neurons of adult mice alters spatial memory. We then examined whether the alteration in NOL observed in Pyk2 KO mice could be mimicked by the targeted deletion of Pyk 2 in the dorsal hippocampus. We used $P t k 2 b^{f / f}$ mice and stereotactically injected adeno-associated virus (AAV) expressing either the recombinase Cre fused to GFP (AAV-GFP/Cre) or GFP alone (AAV-GFP) in the dorsal hippocampus at two injection sites. The AAV serotype (AAV1) and the human synapsin-1 (SYN1 gene) promoter controlling Cre expression preferentially target neuron $s^{27,29}$. Three weeks after the injection, we assessed spatial memory with the NOL test. Twenty-four hours after the first exposure to the objects, $P t k 2 b^{f / f}$ mice injected with AAV-GFP retained their ability to distinguish the moved object (Fig. 2B). In contrast, the mice injected with AAV-GFP/Cre lost this ability (Fig. 2B). The mean difference in time spent exploring NL between AAV-GFP- and AAV-GFP/Cre-injected mice was - 12.9\% (95.0\% CI - 21.5, - 3.41) with a P value of the two-sided permutation t-test of 0.0092 (Supplementary Table 1). This result, in agreement with a previous report ${ }^{21}$, shows that targeted deletion of Pyk2 in the dorsal hippocampus is sufficient to alter performance in the NOL test. This result rules out developmental effects or indirect consequences of Pyk2 deletion in 
A

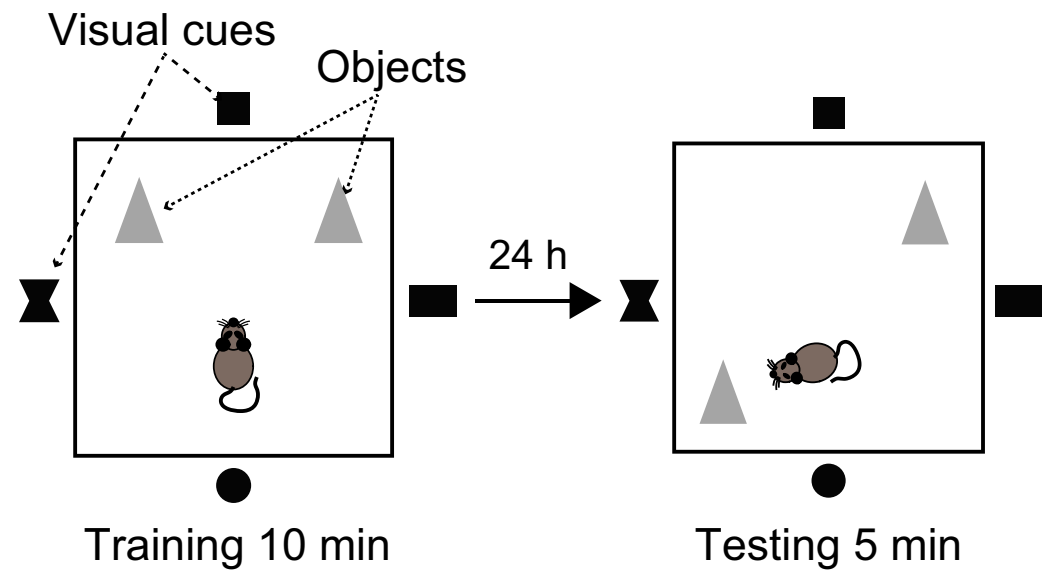

B

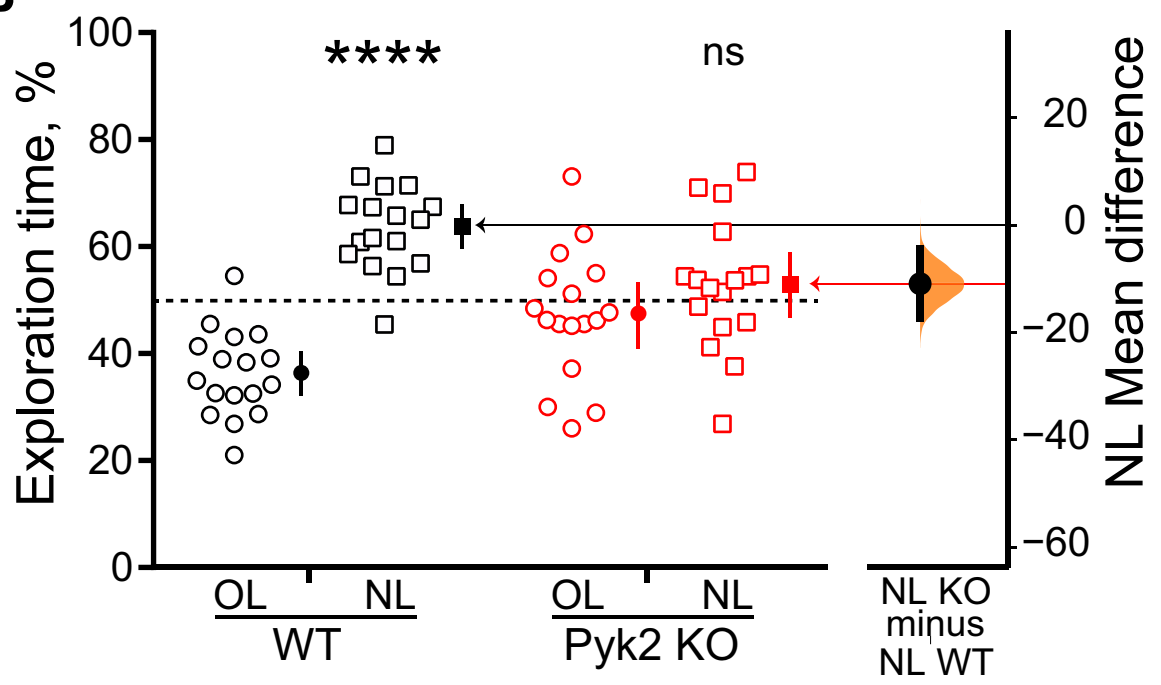

Figure 1. Pyk2 knockout alters novel object location (NOL). (A) Schematic representation of the test principle. After habituation of the mouse to the arena, two identical objects (indicated as grey triangles) are positioned and the animal is allowed to explore them for $10 \mathrm{~min}$ (left panel). Black on white visual cues outside of the arena facilitate spatial location of the objects. One day later the mouse is placed for $5 \mathrm{~min}$ in the same arena in which the location of one of the two objects has been modified. The time spent exploring the displaced object (new location, NL) and the unmoved object (old location, OL) is measured by video tracking. (B) Time spent exploring OL and NL is plotted on the left axis with open symbols for individual mouse values of Pyk2 wild type mice $(n=17)$ and Pyk2 KO mice $(n=17)$. Closed symbols indicate means, ends of vertical bars indicate the $95 \%$ confidence interval (CI), and the horizontal dotted line indicates the chance level (50\%). One-sample t-test (NL exploration time vs. $50 \%$ chance preference) $\mathrm{WT}, \mathrm{t}_{16}=6.99, \mathrm{p}<10^{-4}, \mathrm{KO}, \mathrm{t}_{16}=0.96, \mathrm{p}=0.35$. The mean difference between NL WT and NL KO is shown as Gardner-Altman estimation plot and is plotted on a floating axis on the right as a bootstrap sampling distribution. The mean difference is depicted as a dot; the $95 \% \mathrm{CI}$ is indicated by the ends of the vertical error bar. Comparison of NL WT vs NL KO, unpaired two-tailed $t$ test with Welch's correction, $\mathrm{t}_{28}=3.09, \mathrm{p}=0.0045 .^{* *}, \mathrm{p}<0.01,{ }^{* * *} \mathrm{p}<10^{-4}$, ns, not significant. Statistical results in Supplementary Table 1.

other brain regions or tissues. In addition, because the AAV serotype and the promoter of the viral vector used display cell type specificity, these results also provide information about the location of Pyk2 critical for the behavioral deficit, presumably dorsal hippocampus pyramidal neurons.

CA1 LTP is impaired in Pyk2 KO mice in an induction protocol-dependent manner. In mice used for behavioral evaluation, we then investigated LTP at synapses between Schaffer collaterals and CA1 pyramidal cells. These synapses are the site of one of the best characterized forms of NMDA-dependent synaptic plasticity important for spatial memory ${ }^{30}$. We used acute hippocampal slices from wild type (age range 2.1-4.6 months, mean \pm SEM, 3.0 \pm 0.3 ) and Pyk2 KO (age range 2.0-3.2 months, mean \pm SEM, $2.5 \pm 0.2$ months) mice. The absence of Pyk2 protein was verified by immunoblotting of the hippocampus of KO mice as compared to wild 

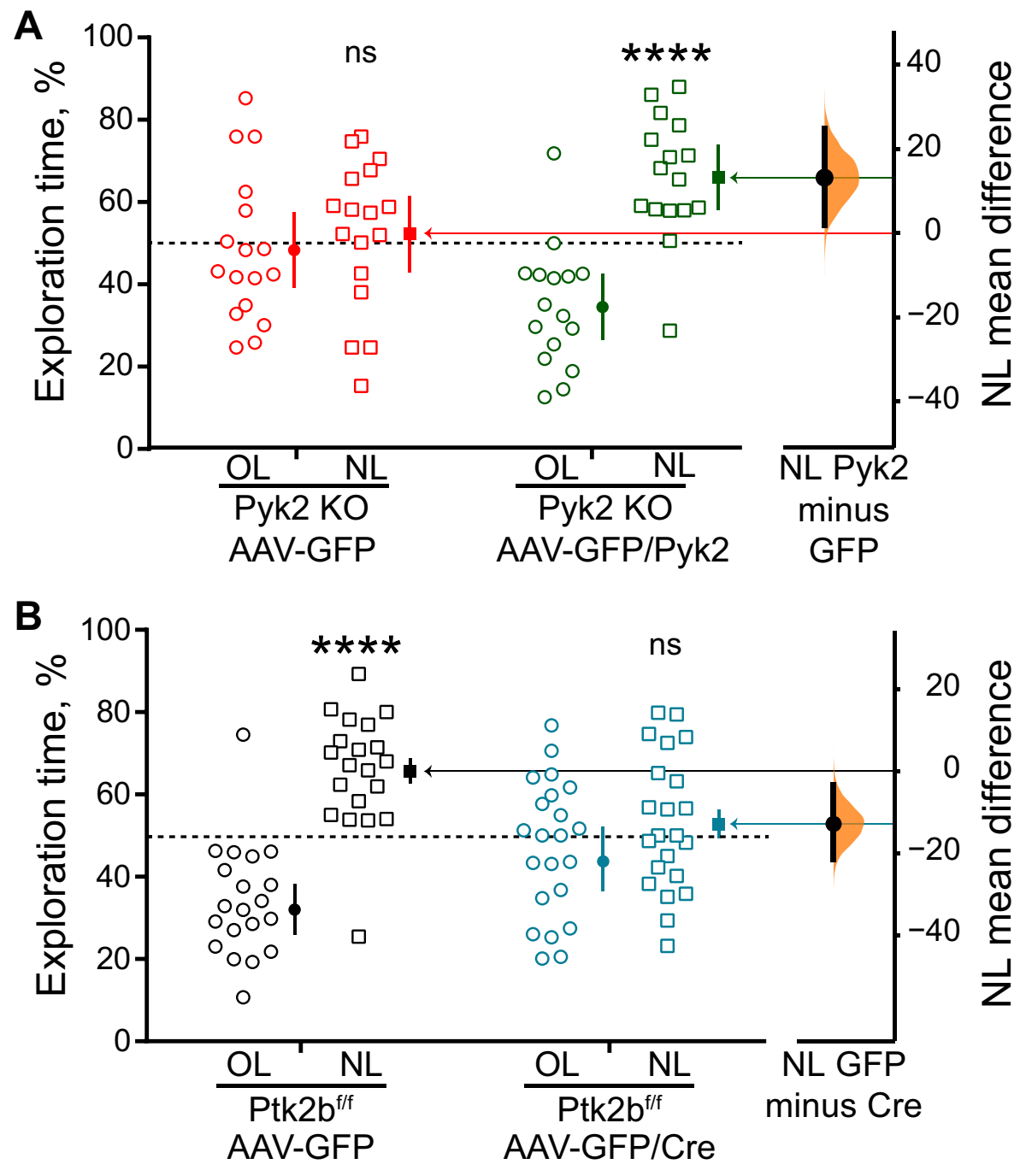

Figure 2. The deficit in NOL in Pyk $2 \mathrm{KO}$ mice is rescued by re-expression of Pyk 2 in the dorsal hippocampus and mimicked by its selective deletion in this region. (A) Pyk2 re-expression rescues NOL. Pyk2 KO male mice were injected bilaterally in dorsal hippocampus CA1 with AAV-GFP $(n=17)$ or AAV-GFP/Pyk2 $(n=16)$. Three weeks later their performance in the NOL test was evaluated and plotted as in Fig. 1. The time spent exploring the object at NL for AAV-GFP-injected mice was not different from random choice for KO AAV-GFP-injected mice (one-sample t-test NL exploration time vs. $50 \%$ chance preference, $\mathrm{t}_{16}=0.45, \mathrm{p}=0.66$ ), whereas it was for KO AAV-GFP/Pyk2-injected mice $\left(\mathrm{t}_{15}=4.22, \mathrm{p}=0.0008\right)$. Comparison of NL KO AAV-GFP vs KO AAV-GFP/ Pyk2, unpaired two-tailed $t$ test with Welch's correction, $\mathrm{t}_{30}=2.40, \mathrm{p}=0.02$. Mean difference in time spent exploring NL between AAV-GFP and AAV-GFP/Pyk2 plotted as in Fig. 1B, two-sided permutation t-test, $\mathrm{p}=0.024$. (B) Targeted conditional deletion of Pyk2 in dorsal hippocampus CA1 alters NOL. Pyk $2^{\mathrm{f} / \mathrm{f}}$ male mice injected bilaterally with either AAV-GFP $(n=20)$ or AAV-GFP/Cre $(n=22)$ were tested for NOL 3 weeks later. One-sample t-test (NL exploration time vs. $50 \%$ chance preference) AAV-GFP-injected mice, $\mathrm{t}_{19}=5.11$, $\mathrm{p}<10^{-4}$, AAV-GFP/Cre-injected mice, $\mathrm{t}_{21}=0.84, \mathrm{p}=0.41$. Mean difference in time spent exploring NL between AAV-GFP and AAV-GFP/Cre plotted as in Fig. 1B, two-sided permutation t-test, $p=0.0092$. In $(\mathbf{A})$ and $(\mathbf{B})$, the horizontal dotted line indicates the chance level (50\%), bars indicate means $+\mathrm{SEM},{ }^{*}, \mathrm{p}<0.05,{ }^{* * *} \mathrm{p}<10^{-4}, \mathrm{~ns}$, not significant. Statistical results in Supplementary Table 1.

type (Fig. 3A). We stimulated Schaffer collaterals and measured fEPSP slopes in CA1. To ensure that the loss of Pyk2 had no influence on the efficiency of stimulation on the Schaffer collaterals or major pre-synaptic effect, we first analyzed the input/output relationship between the fiber volley amplitude (representing the stimulation of the Schaffer collaterals) and the fEPSP slope (representing the post-synaptic response) and found no difference between wild type and Pyk2 KO slices (Fig. 3B).

In these experimental conditions, we induced LTP using two different stimulating protocols, which have been previously used to assess the role of Pyk2 in hippocampal synaptic plasticity with contrasting results ${ }^{21,22}$. The first protocol was a high-frequency stimulation (HFS) protocol (5 times 1-s stimulation at $100 \mathrm{~Hz}$ with intervals of $10 \mathrm{~s}^{21}$ ), and the second protocol was a theta-burst stimulation (TBS) protocol (10 bursts of 4 shocks at $100 \mathrm{~Hz}$ with an interburst interval of $200 \mathrm{~ms}^{22}$ ). When we induced LTP in slices from wild type animals with the HFS protocol, 
we elicited a robust and persistent LTP (Fig. 3C,D). In contrast, when we stimulated slices from Pyk2 KO animals with HFS, we found a significantly reduced LTP (Fig. 3C,D, Supplementary Table 2). The mean difference in average EPSP slope at 50-60 min (expressed as \% of baseline normalized slope) between Pyk2 WT and KO mice was $-22.6 \%$ (95.0\% CI $-39.6,-7.65)$, with a two-sided permutation t-test $\mathrm{p}$ value $=0.0068$ (Fig. 3D, Supplementary Table 2). During the same experimental sessions and using different slices, including some from the same animals as for the HFS protocol, we induced LTP using the TBS protocol described above. With TBS we found no difference in the elicited LTP between slices from wild type or Pyk2 KO animals (Fig. 3E,F, Supplementary Table 3). The mean difference in average EPSP slope at 50-60 min between Pyk2 WT and KO mice was - 2.1\% (95.0\% CI, $-20.5,16.8$ ), with a two-sided permutation t-test $\mathrm{p}$ value $=0.86$ (Fig. 3F, Supplementary Table 3 ). These results obtained with two different types of stimulation in the same mouse line show that the alteration in LTP induced by the absence of Pyk2 depends on the protocol used for inducing synaptic potentiation.

Targeted deletion of Pyk2 in dorsal hippocampus CA1 alters LTP in an induction protocol-dependent manner. We then examined whether loss of Pyk2 induced in adult animals and limited to dorsal hippocampus CA1 region was able to affect synaptic plasticity in acute hippocampal brain slices, as above for the NOL test. We measured fEPSPs in CA1 of hippocampal slices from $P t k 2 b^{f / f}$ animals injected with either AAV-GFP/Cre (age range 2.4-6.4 months, mean \pm SEM, 4.9 \pm 0.6 ) or AAV-GFP (age range 2.4-6.6 months, mean \pm SEM, $4.6 \pm 0.8$ ) and measured the LTP induced by either HFS or TBS. We verified the correct targeting of the stereotaxic injections in each slice on the recording rig (Fig. 4A, left panel and a1-2) and observed that GFP fluorescence was confined to CA1, mostly in the pyramidal layer (Fig. 4A, a3-5). Using only slices in which GFP was visually expressed in CA1, we analyzed the input/output relationship between fiber volley amplitude and fEPSP slope in slices from $P t k 2 b^{f / f}$ mice injected either with AAV-GFP/Cre or AAV-GFP and found no difference (Fig. 4B), supporting the lack of presynaptic effect, as expected by the restriction of viral spread to CA1.

We then induced LTP in different slices with either HFS or TBS, the two protocols described above. We first monitored the fiber volley amplitude over time after HFS and did not observe any major change or difference between AAV-GFP- and AAV-GFP/Cre-injected mice (Fig. 4C), supporting the absence of presynaptic modification. As expected, in slices from $P t k 2 b^{t / f}$ animals injected with AAV-GFP, the HFS protocol induced a robust and persistent LTP (Fig. 4D) at levels comparable to wild type animals (Fig. 3C), showing the lack of effect of the presence of floxed alleles of $P t k 2 b$ gene, virus injection or GFP expression on this parameter. In contrast, LTP induced by HFS was markedly reduced in slices from $P t k 2 b^{\mathrm{f} / \mathrm{f}}$ mice injected with AAV-GFP/Cre (Fig. 4D, Supplementary Table 4). The mean difference in average EPSP slope at 50-60 min (expressed as \% of baseline normalized slope) between slices of GFP- and GFP/Cre-expressing mice was - 46.9\% (95.0\% CI - 74.0, - 22.2), with a two-sided permutation t-test $\mathrm{p}$ value $=0.0064$ (Fig. 4E, Supplementary Table 4). We also examined the effects of the TBS protocol in slices from Pyk2f./f animals injected with AAV-GFP or AAV-GFP/Cre. TBS elicited similar levels of LTP in slices from Ptk2 $b^{t / f}$ animals injected with AAV-GFP/Cre or AAV-GFP (Fig. 4F, Supplementary Table 5). The mean difference in average EPSP slope at 50-60 min between slices of GFP/Cre- and GFP-expressing mice was $-13.1 \%(95.0 \% \mathrm{CI}-45.9,9.07)$, with a two-sided permutation $\mathrm{t}$-test $\mathrm{p}$ value $=0.41$ (Fig. 4G, Supplementary Table 5). These results showed that targeted deletion of Pyk2 in dorsal hippocampus CA1 neurons replicates the effects of constitutive knockout, with a selective deficit in LTP induced with a HFS protocol and no alteration with the TBS protocol. They show that the HFS-induced LTP modification is not the consequence of a developmental or indirect alteration. Moreover because the AAV expression detected by GFP fluorescence was expressed in CA1 neurons, mostly in pyramidal layer, and not in CA3, these results indicate that the alteration in HFS-induced LTP very probably results from a post-synaptic defect in these neurons.

\section{Discussion}

In this study, we provide evidence that the loss of the non-receptor tyrosine kinase Pyk2 in mice alters spatial memory and synaptic plasticity in hippocampal neurons in a selective manner. These results support previous observations indicating a role of Pyk2 in spatial memory and LTP and provide a rationale for explaining the phenotypic differences between reports in different mouse lines, which displayed either deficits in NOL and $\mathrm{LTP}^{21}$ or normal LTP and spatial memory in the Morris water maze ${ }^{22}$. We confirm the alteration in the NOL test as reported by Giralt et al. ${ }^{21}$ but not explored by Salazar et al. ${ }^{22}$. This deficit was rescued by re-expression of Pyk2 in the dorsal hippocampus CA1 region and reproduced by targeted deletion in neurons of this brain area. These findings rule out that the phenotypic deficit results from developmental alterations or indirect effects. In addition, because the viral vector serotype $(\mathrm{AAV} 1)^{27}$ and the promoters used to drive the expression of Pyk2 (mouse Camk2a gene) ${ }^{28}$ and Cre (human SYN1 gene) ${ }^{29}$ are known to preferentially target neurons, this memory deficit is likely to derive from the absence of Pyk2 in neurons where it is abundantly expressed ${ }^{7}$. It is important to underline that the memory deficits in Pyk2 KO mice are only partial with altered NOL (Ref. ${ }^{21}$ and this report), but normal performance in the Morris water maze ${ }^{22}$. The Morris water maze is a classical test with multiple variations that explores spatial memory and is altered by hippocampal lesions or dysfunction ${ }^{31,32}$. The NOL test, which evaluates spatial memory $24 \mathrm{~h}$ after a single exposure, is highly sensitive to and relatively selective for dorsal hippocampal alterations ( $\operatorname{see}^{23}$ for a recent review). We do not know why Pyk2-deficient mice display a deficit in the NOL but not in the water maze test, but it is worth noting that the NOL involves a single training session whereas the water maze protocol used with Pyk2 KO mice included multiple training sessions during several days and may be reinforced by the stressful component of the test ${ }^{33}$. We suggest that the partial impairment of synaptic plasticity in Pyk2 mutant mice is sufficient to alter one-trial NOL performance but can be overcome by repeated training or possibly a stress-linked component. Interestingly, a different one-trial training test, normal novel object recognition (NOR), was reported to be normal in Pyk2 KO mice ${ }^{21,22}$. Although NOL and NOR may appear similar, their sensitivity to interferences is different ${ }^{23}$. NOR involves several brain regions and 
A

Pyk2 WT KO WT KO WT KO WT KO Actin

C

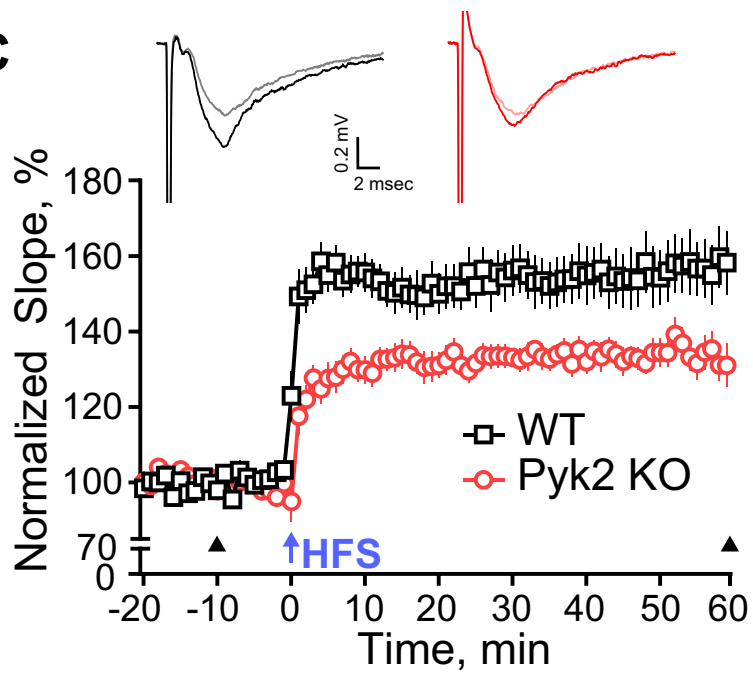

E

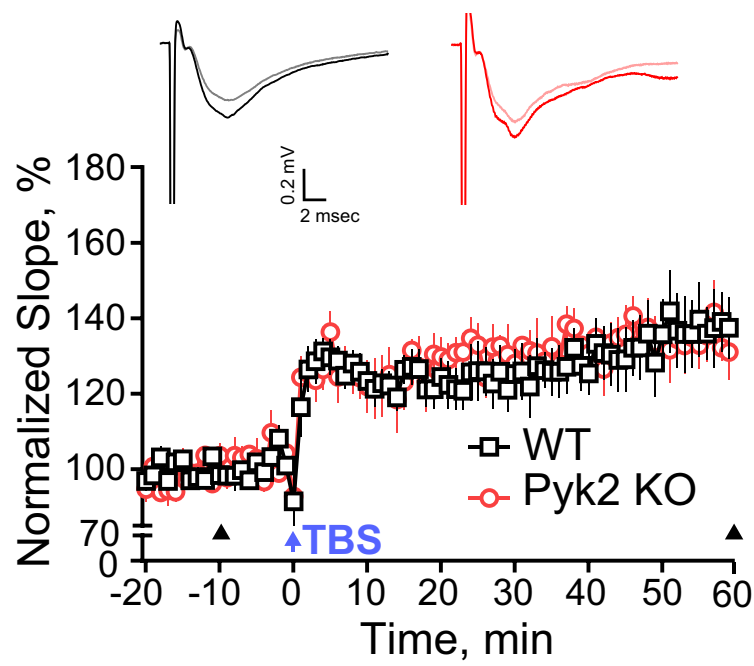

B

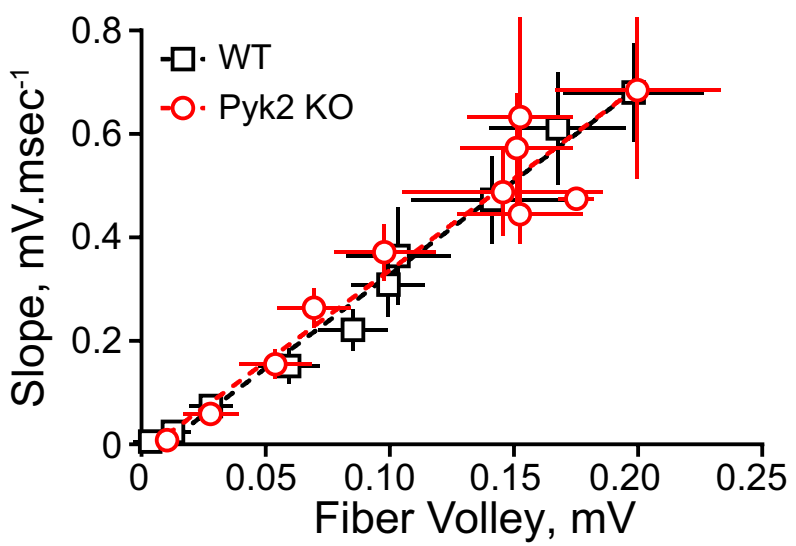

$\mathbf{F}$
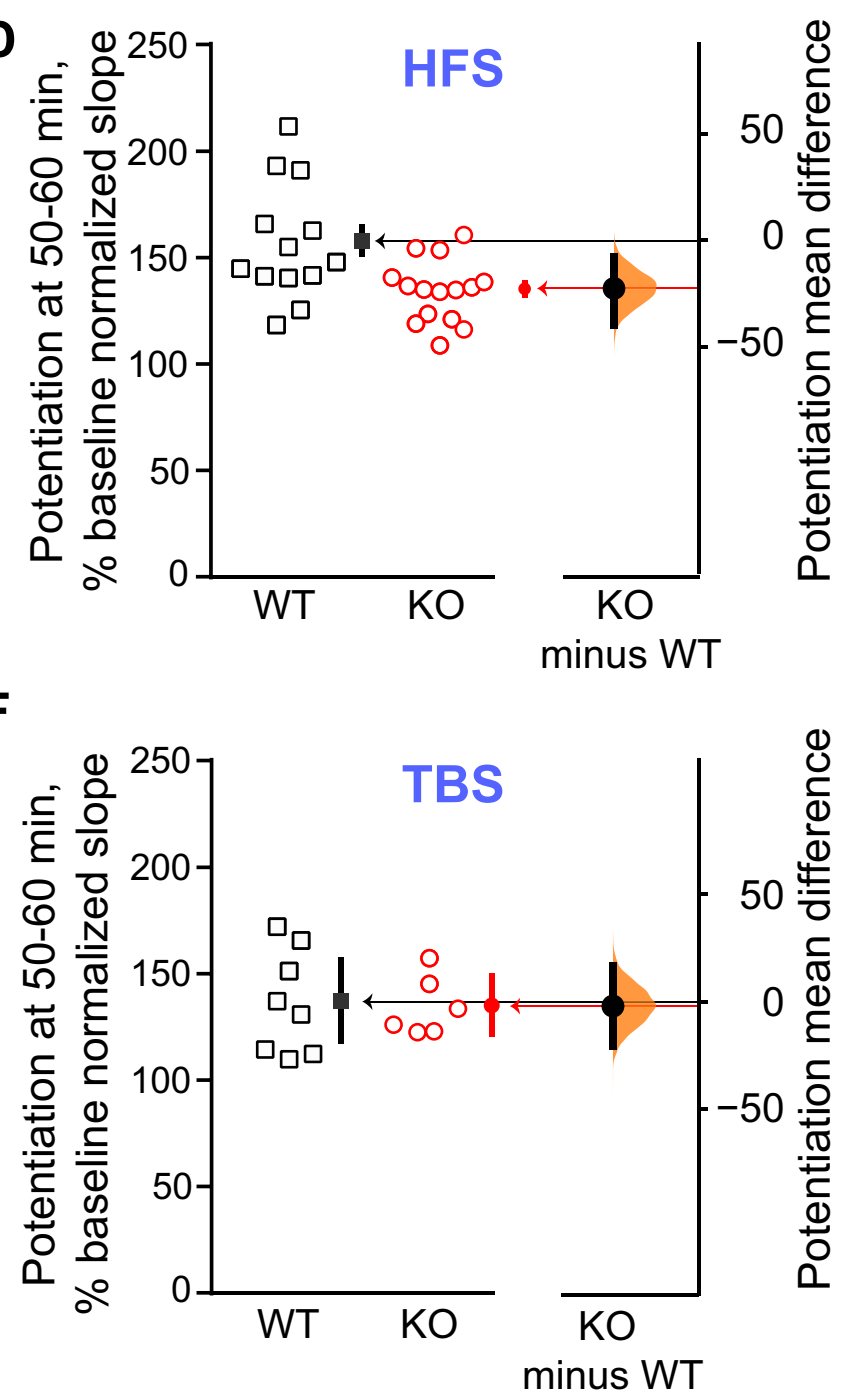
4Figure 3. Selective impairment of LTP at CA1 synapses in Pyk2 KO mice depends on the induction protocol. (A) Hippocampi contralateral to that used for electrophysiology were obtained from $3 \mathrm{KO}$ mice and 3 WT mice and analyzed by immunoblotting for the presence of Pyk2 and actin as a loading control (full-length blots in Supplementary Fig. 1. (B). Schaffer collaterals were stimulated and fEPSP recorded at $32{ }^{\circ} \mathrm{C}$ in CA1 of acute dorsal hippocampus slices from wild type (WT, 8 animals, 13 slices) and Pyk2 KO (7 animals, 15 slices, means \pm SEM). No difference in input/output relationship was observed. (C) High frequency stimulation (HFS)induced LTP is altered in Pyk2 KO mice. Slices were subjected to HFS (five 1-s trains of $100 \mathrm{~Hz}$ stimulation separated by $10 \mathrm{~s}$ intervals, arrow, time 0 ). Slopes of evoked fEPSPs were recorded every minute before and after HFS and expressed as a percentage of slope average during the 20-min baseline (means \pm SEM). Insets at the top: representative traces $10 \mathrm{~min}$ before and $60 \mathrm{~min}$ after HFS stimulation (arrowheads). Two-way repeated measure ANOVA, interaction, $\mathrm{F}_{(79,2054)}=4.63, \mathrm{p}<10^{-4}$, genotype effect, $\mathrm{F}_{(1,26)}=15.76, \mathrm{p}=510^{-4}$. (D) Potentiation during the last $10 \mathrm{~min}$ of recording in (C). Average normalized slope expressed as $\%$ of baseline for each slice 50-60 min after HFS induction, open symbols (left axis), closed symbols, group mean, vertical bars, $95 \%$ confidence interval. Mean difference between WT and Pyk2 KO slices plotted as bootstrap sampling distribution (right axis, mean difference, dot, 95\% confidence interval, vertical bar), two-sided permutation t-test, $\mathrm{p}=0.0092$. (E) Theta burst stimulation (TBS)-induced LTP is not altered in Pyk2 KO mice. Hippocampal slices from WT ( 5 animals, 8 slices) and Pyk2 KO (5 animals, 6 slices) mice were stimulated using a TBS protocol (15 4-pulse bursts at $100 \mathrm{~Hz}$, every $200 \mathrm{~ms}$, arrow). Plot as in (C). Two-way repeated measure ANOVA, interaction, $\mathrm{F}_{(79,948)}=0.63, \mathrm{p}>0.99$, genotype effect, $\mathrm{F}_{(1,12)}=0.10, \mathrm{p}=0.75$. $(\mathrm{F})$ Potentiation at the end of the recording in $(\mathbf{E})$, expressed as in (D). Two-sided permutation t-test, $\mathrm{p}=0.855$. In (D) and (E), ${ }^{\star} \mathrm{p}<0.05,{ }^{* * *} \mathrm{p}<0.001$. Detailed statistical analyses in Supplementary Tables $2(\mathrm{C}-\mathrm{D})$ and $3(\mathrm{E}-\mathrm{F})$.

neurotransmitter systems ${ }^{34}$. Aggleton and Nelson conclude that "transient disruptions of dorsal hippocampal activity and plasticity are sufficient to impair NOL, though the same manipulations spare NOR" 23 . The selective deficit in Pyk2 is likely to be compensated by other mechanisms, including involvement of other brain regions, when memory is tested in NOR but not in NOL. These results highlight that various commonly used behavioral tests explore different aspects of the memory processes and that a selective molecular perturbation can alter some of them but not others.

The selectivity of Pyk2 requirement in hippocampal function is also highlighted by its implication in synaptic plasticity. Several reports using entirely in vitro approaches provided indications of a role of Pyk 2 in LTP at hippocampal synapses. Huang and colleagues carried out patch-clamp studies in acutely dissociated CA1 pyramidal neurons and found that Pyk2 in the patch pipette potentiated NMDA currents ${ }^{10}$. The presence of kinase-dead Pyk2 in the pipette prevented the LTP induced by a tetanic stimulation consisting of two 500-ms trains of $100 \mathrm{~Hz}$ stimuli at a 10-s interval ${ }^{10}$. Bartos and colleagues also investigated LTP in acute hippocampal slices using whole cell patch clamp and tetanic stimulation with two $500-\mathrm{ms} 100 \mathrm{~Hz}$ trains separated by $10 \mathrm{~s}^{2}$. They reported that LTP induction was prevented when the patch pipette contained the SH3 domain of PSD-95 that binds Pyk2 (GST-SH3) or the Pyk2 sequence with which this SH3 domain interacts (GST-Pyk2 [671-875]). The authors concluded that Pyk2 interaction with PSD-95 was necessary for the induction of LTP in these conditions, presumably allowing Pyk2 synaptic clustering and activation ${ }^{2}$. A third report investigated synaptic plasticity in hippocampal slices in organotypic culture with whole cell patch clamp ${ }^{20}$. The authors used a different type of protocol to induce LTP by pairing a stimulation consisting of 200 pulses at $3 \mathrm{~Hz}$ with postsynaptic depolarization to $0 \mathrm{mV}$ and found no effect of Pyk2 down-regulation with shRNA ${ }^{20}$. Divergent results were also reported in studies with mutant mice. LTP induced in hippocampal slices from Pyk2 KO mice at Schaffer collaterals synapses on CA1 pyramidal cells by 5 times repeated 1-s tetanus at $100 \mathrm{~Hz}$ with an interval of $10 \mathrm{~s}$ was blocked $^{21}$. In contrast, in a different Pyk2 KO mouse line, LTP induced at the same synapses by a thetaburst stimulation (TBS) protocol (10 bursts of 4 shocks at $100 \mathrm{~Hz}$ with an interburst interval of $200 \mathrm{~ms}$ ) was not altered $^{22}$. Here we compared these two protocols of LTP induction in the same Pyk2 KO mouse line, including in different slices from the same mice and found that the LTP induced by HFS was altered whereas LTP induced by TBS was not modified. We carried out the same experiments in $P t k 2 b^{\mathrm{f} / \mathrm{f}}$ mice injected with AAV-GFP/Cre in the dorsal hippocampus and observed that HFS-induced LTP was impaired whereas TBS-induced LTP was unchanged in comparison to AAV-GFP-injected mice. Thus, in two different mouse models we replicated the previous findings of the two different groups, providing evidence that the difference in their results was linked to the use of different LTP induction protocols. Both direct manipulation of Pyk2 in hippocampal neurons $\mathrm{s}^{2,10}$ or complete or targeted Pyk2 KO (Giralt et al. ${ }^{21}$ and present report) alter HFS-induced LTP, whereas LTP induced by TBS (Salazar et al..$^{22}$ and present report) or by a related stimulation protocol ${ }^{20}$ is not altered. Interestingly it was reported that HFS-induced LTP was also impaired in heterozygous Pyk2-KO mice ${ }^{21}$, suggesting a dosage sensitivity. In the present work we did not further explore this point since we used homozygous constitutive Pyk2 KO mice and in the mice with virally-induced deletion it was not possible to know whether the deletion was homozygous in all targeted cells.

The importance of the stimulation protocol is also apparent in the retrospective comparison of other studies on the role of tyrosine kinases in synaptic plasticity. The inhibitory effects of tyrosine kinase inhibitors on LTP at CA1 synapses were observed with HFS ${ }^{17,35}$. Grant et al. investigated the hippocampal LTP at CA1 synapses in four non-receptor tyrosine kinase KO lines, 3 SFKs (Fyn, Src, and Yes), and the structurally more distant $\mathrm{Abl}^{36}$. They observed a deficit in Fyn KO mice but not in the other mouse lines, and only when they used two trains of tetanic stimulation trains $(1-\mathrm{s}$ trains at $100 \mathrm{~Hz})$ with an intertrain interval of $20 \mathrm{~s}^{36}$. In contrast they did not observe any alteration when LTP was induced by pairing 40 low frequency $(1 \mathrm{~Hz})$ stimulations with postsynaptic depolarization ${ }^{36}$. This protocol is reminiscent of the one that did not reveal any LTP alteration in Pyk2 knockdown slices (200 pulses at $3 \mathrm{~Hz}$ paired with postsynaptic depolarization), as mentioned above ${ }^{20}$. Taken 
Figure 4. Targeted conditional deletion of Pyk2 in the dorsal hippocampus results in a selective inductiondependent impairment of LTP at CA1 synapses. (A) $P t k 2 b^{\mathrm{f} / \mathrm{f}}$ mice were injected bilaterally at 2 sites in dorsal hippocampus CA1 with AAV-GFP/Cre or AAV-GFP (top left panel). Successful expression was evaluated with fluorescence microscopy on slices prior to electrophysiological recordings (a1, bright field, a2, GFP fluorescence, a3 higher magnification of a 2 (a1-3 are from the same slice, a 4 and a 5 from different mice). Scale bar, a1-2, $500 \mu \mathrm{m}$, a3-5 $200 \mu \mathrm{m}$. (B-E) fEPSP were recorded as in Fig. 3, in Pyk2 ${ }^{\mathrm{f} / \mathrm{f}}$ mice injected with AAVGFP (4 animals, 8 slices) or AAV-GFP/Cre (4 animals, 7 slices). (B, C) No difference was observed in input/ output relationship $(\mathbf{B}$, means \pm SEM) or time course of fiber volley amplitude $(\mathbf{C})$ normalized to the mean before HFS. (D) HFS-induced LTP is altered in mice with a Pyk2 deletion in dorsal hippocampus (plot as in Fig. 3B). Insets at the top: representative traces $10 \mathrm{~min}$ before and $60 \mathrm{~min}$ after HFS stimulation (arrowheads). Two-way repeated measure ANOVA, interaction, $\mathrm{F}_{(79,1027)}=5.13, \mathrm{p}<10^{-4}$, Cre effect, $\mathrm{F}_{(1,13)}=19.83, \mathrm{p}=0.004$. (E) Potentiation during the last $10 \mathrm{~min}$ of recording in (D) expressed and plotted as in Fig. 3D, two-sided permutation t-test, $\mathrm{p}=0.0064$. (F) TBS-induced LTP is not altered by selective deletion of Pyk2 in the dorsal

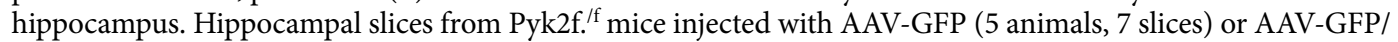
Cre (4 animals, 7 slices) mice were stimulated and time-course of normalized slopes of fEPSPs recorded and plotted as in Fig. 3E before and after TBS (time 0). Insets at the top: representative traces 10 min before and $60 \mathrm{~min}$ after TBS stimulation (arrowheads). Two-way repeated measure ANOVA, interaction, $\mathrm{F}_{(79,948)}=0.89$, $\mathrm{p}=0.73$, Cre effect, $\mathrm{F}_{(1,12)}=0.30, \mathrm{p}=0.59$. $(\mathbf{G})$ Potentiation during the last $10 \mathrm{~min}$ of recording in $(\mathbf{F})$, expressed as in $(\mathbf{E})$. Two-sided permutation t-test, $\mathrm{p}=0.41$. Detailed statistical analyses in Supplementary Tables 4 (D-E) and $5(\mathrm{~F}-\mathrm{G})$.

together these results indicate that the role of non-receptor tyrosine kinases Pyk2 and Fyn at synapses between Schaffer collaterals and CA1 pyramidal neurons is limiting for specific types of plasticity and can be overcome in other conditions.

Why is the role of Pyk2, and presumably of SFKs, important for HFS-induced LTP and not TBS-induced LTP? Previous work from many laboratories provided evidence that HFS-induced LTP results from the pronounced increase in $\mathrm{Ca}^{2+}$-influx through NMDAR $\left(\right.$ review in $^{30}$ ). TBS, also implicates NMDAR activation, but also involves regulation of GABA release $\left(\right.$ review $\mathrm{in}^{37}$ ) and its late phase specifically requires the activation of cAMP-dependent protein kinase ${ }^{38-40}$, illustrating the differential contribution of various signaling pathways to different aspects of synaptic plasticity. Several reports show that Pyk2 can modulate the intensity of NMDAR currents ${ }^{10}$ through the recruitment of SFKs $\left(\mathrm{see}^{13}\right)$. The converging evidence showing the importance of SFKs and Pyk2 in HFS-induced LTP indicates that these tyrosine kinases are specifically required for this type of synaptic plasticity. The activation of Pyk2 and its links with SFKs including Fyn is depicted in Fig. 5. Because tyrosine phosphorylation decreases endocytosis of NMDAR (review in ${ }^{41}$ ), one hypothesis to explain the role of Pyk 2 and SFKs is that they could oppose this endocytosis during intense activation, as suggested by the role of Pyk2 in enhancing NMDAR currents ${ }^{10}$ and surface expression ${ }^{19}$, and thereby maintain sufficient $\mathrm{Ca}^{2+}$ influx. Our data show the consequences of the absence of Pyk2 which could be linked to the absence of its tyrosine kinase activity as suggested by results with kinase-dead mutants ${ }^{10}$, or possibly of some other properties, such as protein-protein interaction. It will be interesting to explore these aspects by using acute and specific pharmacological inhibition of Pyk2 tyrosine kinase activity.

It is surprising that the role of Pyk2 in synaptic plasticity is revealed by HFS-induced LTP, whereas its role in memory is apparent for spatial memory after a single brief training protocol. However, HFS-induced LTP explores synaptic properties in conditions that are not physiological and there is no simple correspondence between specific forms of LTP in slices and behavioral responses ${ }^{42}$. The link between the two alterations observed in the absence of Pyk2 is unclear. In addition, other changes including intrinsic plasticity are likely to be important for memory ${ }^{43}$ in which the role of Pyk2 remains to be explored.

In conclusion, the present work clarifies the role of Pyk2 in synaptic plasticity and memory. It confirms that in the absence of Pyk2 in the dorsal hippocampus, a one-trial test of spatial memory, NOL, is impaired and shows that this impairment can be rescued by re-expression of Pyk2. The lack of alteration of other tests ${ }^{21,22}$ indicates that the consequences of Pyk2 deficit can be overcome by repeated training (Morris water maze) or recruitment of extra-hippocampal mechanisms (novel object recognition). Our results also demonstrate the dissociation between the role of Pyk2 in LTP, which is necessary for HFS-induced LTP but not TBS-induced LTP in CA1. This dissociation is in agreement with previous reports on the role of non-receptor tyrosine kinases at those synapses. Our findings clarify previous contradictions between results of several laboratories and provide a better understanding of the role of non-receptor tyrosine kinases in synaptic plasticity. Future work is needed to elucidate the precise molecular mechanisms underlying the selective requirement of Pyk2 for specific aspects of memory and synaptic plasticity.

\section{Material and methods}

Mice. All experiments were carried out in male mice. We used $P t k 2 b^{-1-}$ (Pyk2 KO) mice and mice with floxed Pyk2 alleles $\left(P t k 2 b^{\mathrm{f} / \mathrm{f}}\right)$ on a $\mathrm{C} 57 \mathrm{Bl} / 6$ background. KO mice were bred by mating $P t k 2 b^{+/-}$heterozygous mice. The $P t k 2 b^{-/-}(\mathrm{KO})$ and $P t k 2 b^{+/+}$(wild type) used for experiments originated from these breeding pairs. Mice were housed under conditions of controlled temperature $\left(23^{\circ} \mathrm{C}\right)$ and illumination $(12$-h light/12-h dark cycle, ZT0=7:00 a.m.). Animal procedures were authorized by and performed in accordance with the animal care committee's regulations. The study was carried out in compliance with the ARRIVE guidelines (https://arriv eguidelines.org) and in accordance with guidelines of Declaration of Helsinki and NIH (1985-revised publication no. 85-23, European Community Guidelines), and French Agriculture and Forestry Ministry guidelines 
A

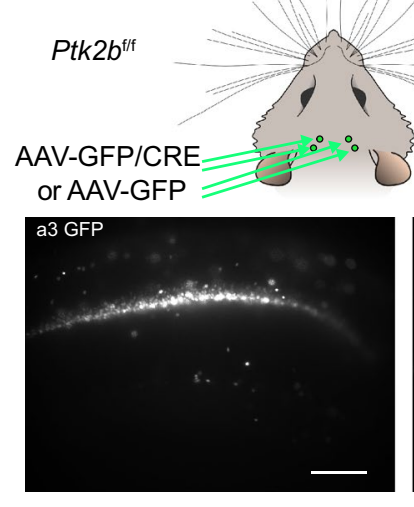

B

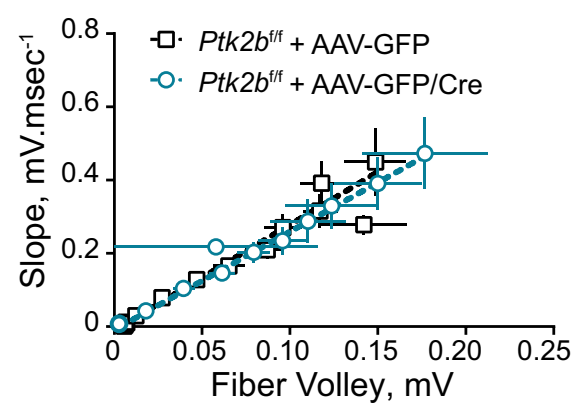

D

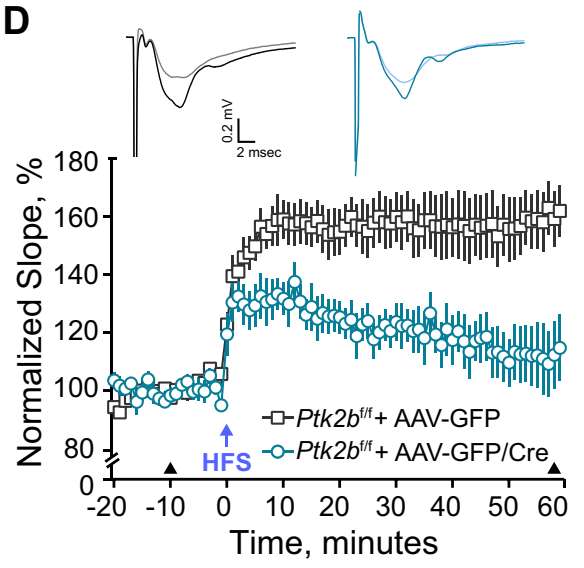

$\mathbf{F}$
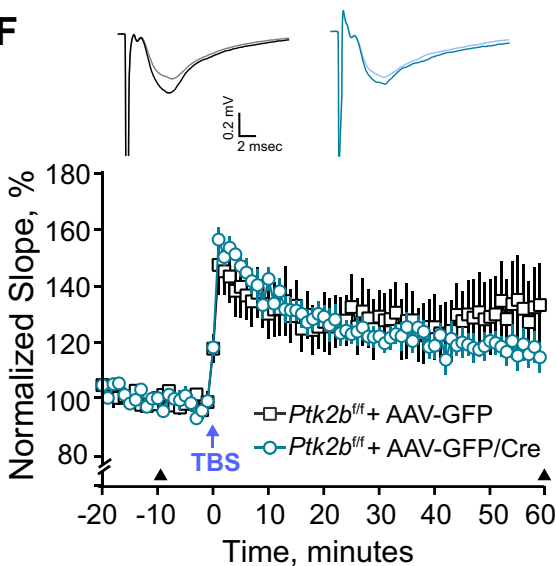
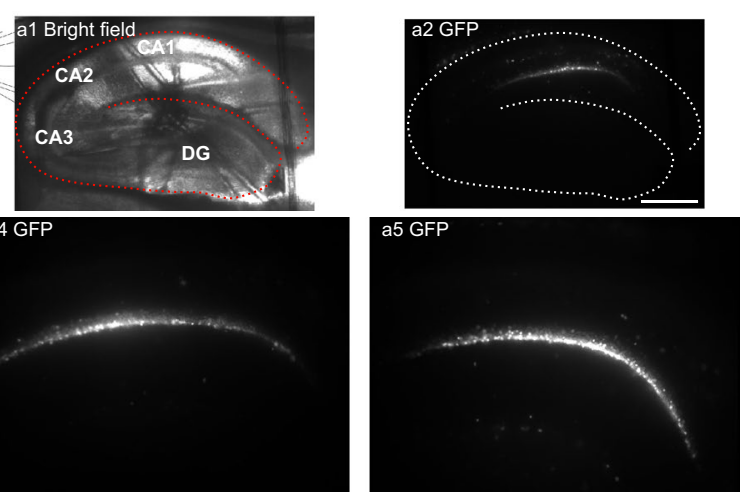

C

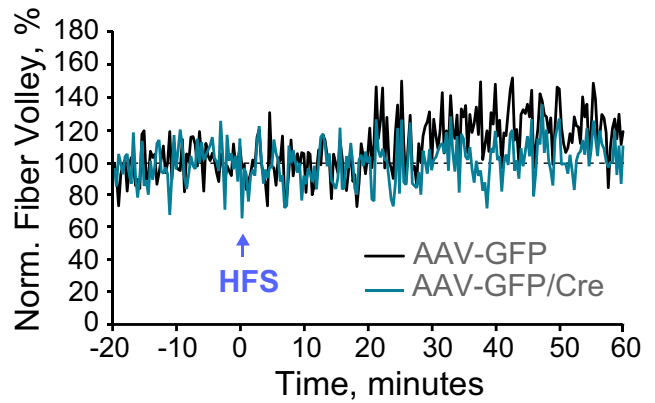

E

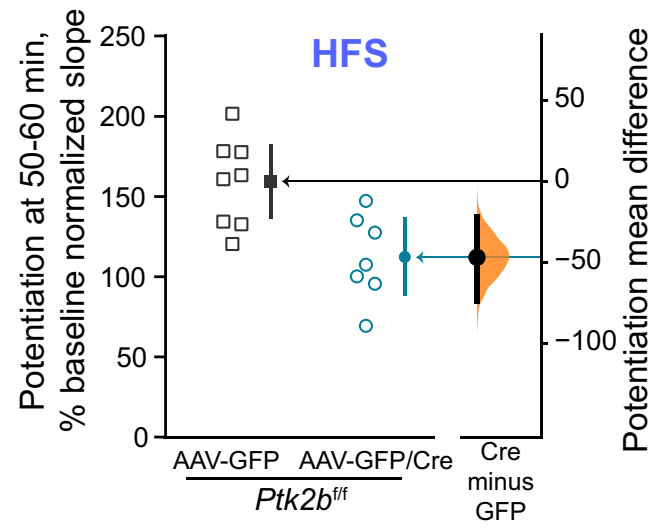

G

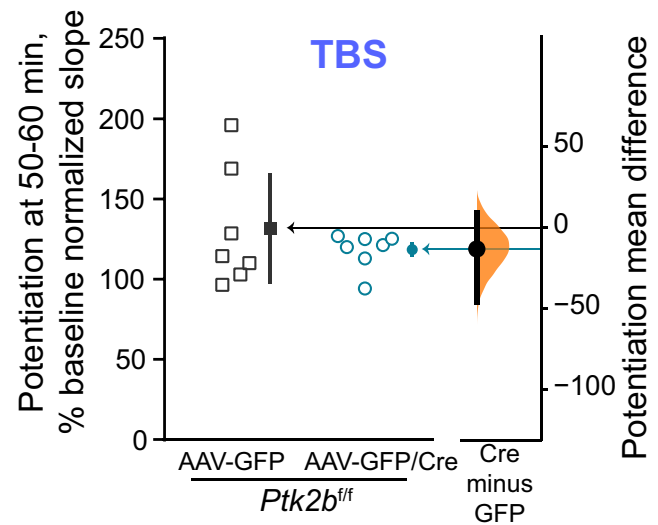




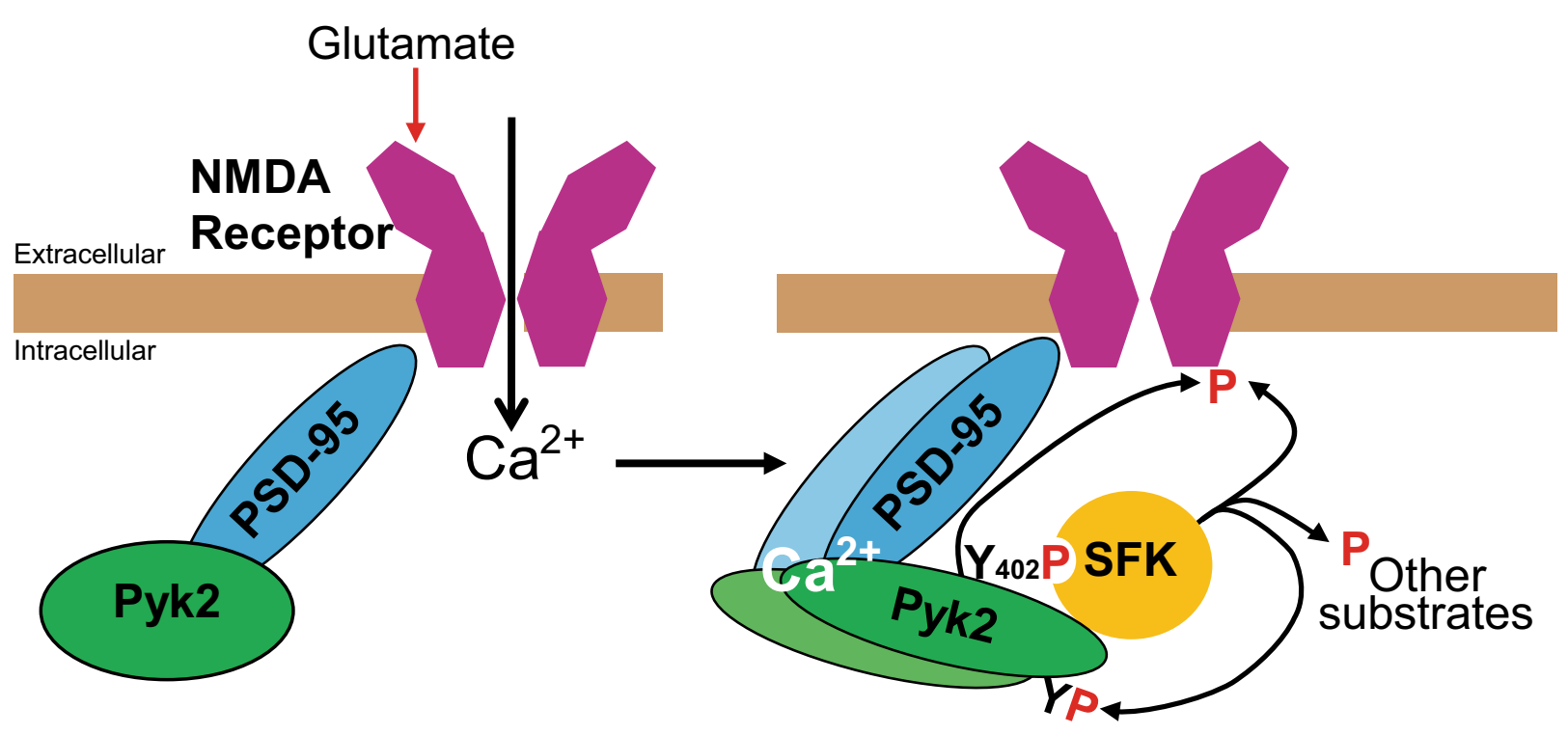

Figure 5. Schematic representation of Pyk 2 activation at synapses. Pyk 2 is associated with NMDA receptors (NMDAR) through its interactions with PSD proteins such as PSD-95. Following $\mathrm{Ca}^{2+}$ entry through activated NMDAR, Pyk2 is activated, presumably by intermolecular autophosphorylation promoted by interaction of $\mathrm{Ca}^{2+}$ and calmodulin (not shown) with Pyk2 and/or associated PSD-95. Autophosphorylated Tyr-402 in Pyk2 provides a binding site for the Src-homology domain 2 (SH2) of Src-family kinases (SFKs) such as Fyn or Src. SFKS also interact with Pyk2 through their SH3 domain (details not depicted in the cartoon). This results in the activation of SFKs which can phosphorylate various tyrosine residues in Pyk2 and increase its kinase activity. SFKs and presumably Pyk2 phosphorylate NMDAR on tyrosine, which increases NMDA currents, at least in part by increasing NMDAR at the cell surface. In addition, SFKs and Pyk 2 can phosphorylate other substrates and activate various signaling pathways that have not yet been fully characterized at the post-synaptic level. See the text for references.

for handling animals (decree 87849, license A 75-05-22), with approval of the ethical committee (agreement APAFIS\#8861-2016111620082809).

Viral constructs and stereotaxic injections. For specific deletion of Pyk2 in the dorsal hippocampus, 6-10 week-old male Pyk2 ${ }^{\mathrm{f} / \mathrm{f}}$ mice were stereotactically injected either with AAV expressing eGFP fused with the recombinase Cre, including a Woodchuck hepatitis virus posttranscriptional regulatory element (WPRE) to enhance expression, under the control of the human synapsin-1 gene promoter (AAV-GFP/Cre, 105540AAV1, pENN.AAV1.hSyn.HI.eGFP-Cre.WPRE.SV40, Addgene, Massachusetts, USA) or with AAV expressing only GFP (AAV-GFP, AV-9-PV1917, AAV9.CamKII0.4.eGFP.WPRE.rBG from Perelman) as control. For reexpression of Pyk2 in the dorsal hippocampus of Pyk2 KO mice, the AAV used was AAV1-CamKIIa (0.4)-GFP2AmPTK2B, polycistronically expressing GFP and Pyk2 separated by a 2A site (AAV-GFP/Pyk2, Vector Biolabs Malvern, PA, USA) or, as control, AAV-GFP, as above. Mice were anesthetized by an intraperitoneal injection of ketamine $15 \mathrm{mg} \mathrm{kg}^{-1}$ and xylazine $3 \mathrm{mg} \mathrm{kg}^{-1}$, and placed in a stereotaxic frame (Kopf Instruments, Sweden). The hippocampus was targeted bilaterally using a 32-gauge needle connected to a 1-ml syringe (NeuroSyringe, Hamilton). Two injections were done on each side, at different locations in the dorsal hippocampus $\mathrm{CA} 1$ region with the following coordinates from the bregma (millimeters): $\mathrm{AL}-1.8, \mathrm{ML} \pm 1.2, \mathrm{DV}-1.5$, and $\mathrm{AL}$ $-2.3, \mathrm{ML} \pm 1.5, \mathrm{DV}-1.5$. AAVs were delivered at a rate of $0.1 \mu \mathrm{min}^{-1}$ for $5 \mathrm{~min}(0.5 \mu \mathrm{l}$ per injection site $)$. After the viral particles were infused, the needle was kept in place for 5 additional minutes before removal. After the procedure, the incision was closed with surgical sutures, and mice were placed in a heated cage until they recovered from anesthesia. After $2 \mathrm{~h}$ of monitoring, mice were returned to their home cage for 3 weeks before starting analyses of behavior and electrophysiology. To reduce suffering, mice received a subcutaneous injection of a non-steroidal anti-inflammatory drug (flunixin, $4 \mathrm{mg} / \mathrm{kg}$ ) just after the surgery and on the next day, when necessary. Behavioral experiments were carried out 3 weeks later in all animals that had been stereotactically injected.

Behavioral phenotyping. For novel object location (NOL) test we used an open-top arena $(45 \times 45 \times 45 \mathrm{~cm})$ under dim light (60-70-lx intensity). Mice (3-5-month old) were first habituated to the arena for 2 days for 15 min per day. To allow mice to identify their position, 4 different visual cues were placed around the arena: one circle, one square, one rectangle and one with the shape of an hourglass, all solid black on a white background. On day 3, two identical objects (Eiffel tower bronze figurines, painted in gray, about $9 \mathrm{~cm}$-high and $3 \mathrm{~cm}$ in diameter) were placed in the arena and mice were left to explore the arena for $10 \mathrm{~min}$. Twenty-four hours later, one of the objects was moved from its original location to the diagonally opposite corner and mice were allowed to explore the arena for $5 \mathrm{~min}$ (Fig. 1A). The mice were continuously recorded with a video tracker (Viewpoint, Newcastle Upon Tyne, UK) placed above the arena, and the percentage of time exploring the displaced object 
(new location, NL) and the unmoved object (old location, OL) was compared. Object exploration was defined as mice actively sniffing or touching the object with their nose or forepaws. Quantification was done manually with a stopwatch.

Slice preparation. Male 2-6 month-old mice were anaesthetized by an intraperitoneal injection of ketamine $15 \mathrm{mg} \mathrm{kg}^{-1}$ and xylazine $3 \mathrm{mg} \mathrm{kg}^{-1}$. Following the achievement of full anesthesia and analgesia, mice where immobilized and a sagittal thoracic incision was made in order to expose the heart. An ice-cold low $\mathrm{Ca}^{2+} /$

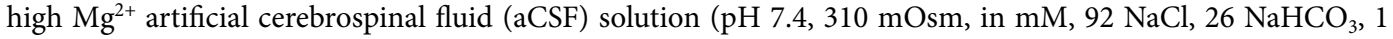
$\mathrm{NaH}_{2} \mathrm{PO}_{4}, 3 \mathrm{KCl}, 12.5$ glucose, 20 Hepes, $0.2 \mathrm{CaCl}_{2}, 7 \mathrm{MgCl}_{2}$,) was intracardially perfused for $2-3$ min until the liver acquired a pale color. The mouse was then swiftly decapitated, the brain was removed and quickly placed in a custom-made $3 \mathrm{D}$-printed brain matrix to rapidly excise the hemispheres with a $45^{\circ}$-angle, necessary for producing hippocampal transversal slices ${ }^{44}$. Each hemisphere was placed on a Petri dish, hold in place with magnets on the vibrating slicer cutting block (Thermo Fisher, USA) and filled with ice-cold low $\mathrm{Ca}^{2+} / \mathrm{high}^{\mathrm{Mg}} \mathrm{aCSF}^{2+}$ solution with constant carbogen $\left(95 \% \mathrm{O}_{2} / 5 \% \mathrm{CO}_{2}\right)$ bubbling. Slices $(350 \mu \mathrm{m}$-thick $)$ were cut $\left(0.08 \mathrm{~mm} \mathrm{~s}^{-1}\right.$, blade vibration, $60 \mathrm{~Hz}$ and $1 \mathrm{~mm}$ ) and individually moved in NMDG-aCSF solution ( $\mathrm{pH} \mathrm{7.4,} 310 \mathrm{mOsm}$, in mM, 93 $\mathrm{N}$-methyl-D-glucamine [glucamine, NMDG], $30 \mathrm{NaHCO}_{3}, 1.2 \mathrm{NaH}_{2} \mathrm{PO}_{4}, 2.5 \mathrm{KCl}, 25$ glucose, $20 \mathrm{Hepes}, 7$ ascorbate, 4 pyruvate, $0.2 \mathrm{CaCl}_{2}, 7 \mathrm{MgCl}_{2}$ (adapted from ${ }^{45}$ ) for $10 \mathrm{~min}$ at $32{ }^{\circ} \mathrm{C}$ for recovery. Slices were then moved to a custom-made $3 \mathrm{D}$-printed interface chamber ${ }^{44}$ containing a modified aCSF solution ( $\mathrm{pH} 7.4,310 \mathrm{mOsm}$, in $\mathrm{mM}, 92 \mathrm{NaCl}, 26 \mathrm{NaHCO}_{3}, 1 \mathrm{NaH}_{2} \mathrm{PO}_{4}, 3 \mathrm{KCl}, 12.5$ glucose, $20 \mathrm{Hepes}, 2 \mathrm{CaCl}_{2}, 1.3 \mathrm{MgCl}_{2}$ ) with constant carbogen $\left(95 \% \mathrm{O}_{2} / 5 \% \mathrm{CO}_{2}\right)$ bubbling and allowed to rest for $2 \mathrm{~h}$ at room temperature.

Electrophysiology. After the 2-h rest, each slice was placed in a custom-made 3D-printed perfusion chamber ${ }^{44}$, with constant flow of recording artificial CSF solution (aCSF, pH 7.4, $310 \mathrm{mOsm}$, in mM, $124 \mathrm{NaCl}$, $26 \mathrm{NaHCO}_{3}, 1 \mathrm{NaH}_{2} \mathrm{PO}_{4}, 3 \mathrm{KCl}, 12.5$ glucose, $5 \mathrm{Hepes}, 2.5 \mathrm{CaCl}_{2}, 1 \mathrm{MgCl}_{2}$ ) at $32{ }^{\circ} \mathrm{C}$ with constant carbogen $\left(95 \% \mathrm{O}_{2} / 5 \% \mathrm{CO}_{2}\right)$ bubbling and let equilibrate for $30 \mathrm{~min}$. The whole recording was carried out in this aCSF at $32^{\circ} \mathrm{C}$. In the experiments with AAV transduction the expression of GFP was visually verified and only the slices in which GFP expression was clearly visible in and restricted to CA1 were used. The stimulating and recording electrodes were made of silver wire encased in borosilicate glass pulled pipettes (pipette resistance: stimulating electrode 1-1.5 M $\Omega$, recording electrode 5-6 M $\Omega$ ) filled with aCSF. The electrodes were positioned in the hippocampal CA1 area, in the middle of the stratum radiatum, $300 \mu \mathrm{m}$ apart from each other. Stimulating currents were delivered using and external ISO-Flexi stimulator (A.M.P.I., Israel) while field excitatory post-synaptic potential (fEPSP) acquisition was performed with a 700B amplifier (Molecular Devices, USA) connected to a 1440a digitizer (Molecular Devices, USA) using the Clampex 10 acquisition software (Molecular Devices, USA). To stimulate the Schaffer collaterals coming from the CA3 area, the stimulating electrode (positioned proximal, towards CA3) delivered a single depolarizing current pulse. A recording electrode was placed distal (towards the subiculum) within the stratum radiatum to record the fEPSPs elicited by Schaffer collaterals stimulation. The current amplitude was set to $50 \%$ of the current that elicited the maximal fEPSP amplitude (current injection 0.6-1.2 $\mu \mathrm{A})$. Stimulating pulses $(0.1 \mathrm{~ms})$ were delivered every $20 \mathrm{~s}(0.033 \mathrm{~Hz})$ and the resulting fEPSPs were recorded. After collecting a 20-min baseline, LTP was induced with either a high-frequency stimulation (HFS) protocol consisting of 5 tetanic bursts of $1 \mathrm{~s}$ at $100 \mathrm{~Hz}$ separated by 10 -s intervals or with a theta-burst stimulation (TBS) consisting of 15 bursts of 4 spikes at $100 \mathrm{~Hz}$ separated by $200-\mathrm{ms}$ intervals. After the induction, fEPSPs were monitored during the next $60 \mathrm{~min}$ and LTP was quantified by analyzing the slope of the activation phase. Only slices with stable recordings throughout the experiment and, in the case of those injected with AAV, only slices with good GFP expression in CA1, were included in the final analyses.

Immunoblotting. Tissue samples were sonicated in $10 \mathrm{~g} \mathrm{l}^{-1} \mathrm{SDS}$ and placed at $100^{\circ} \mathrm{C}$ for $5 \mathrm{~min}$. Extracts (15 $\mu \mathrm{g}$ protein) were separated by SDS-PAGE and transferred to nitrocellulose membranes (GE Healthcare, Chicago, IL, USA). Membranes were blocked in TBS (150 mM NaCl, $20 \mathrm{mM}$ Tris-HCl, pH 7.5) with $0.5 \mathrm{ml} \mathrm{L}^{-1}$ Tween 20 TBS-T) with $30 \mathrm{~g} \mathrm{l}^{-1}$ BSA. Immunoblots were probed with rabbit polyclonal antibodies for Pyk2 (\#P3902, Sigma-Aldrich) diluted 1:1000. Membranes were incubated with the primary antibody overnight at $4{ }^{\circ} \mathrm{C}$ by shaking in TBS. After several washes in TBS-T, blots were incubated with secondary anti-rabbit IgG DyLight $^{\text {Th }} 680$ conjugated antibodies (1:10,000; Rockland Immunochemicals, Pottstown, PA, USA). Secondary antibody binding was detected by Odyssey infrared imaging apparatus (Li-Cor Inc., Lincoln, NE, USA).

Statistical analyses. Data were analyzed with d'Agostino-Pearson and Shapiro-Wilk normality tests. When their distribution did not significantly deviate from normal distribution and the number of replicate was sufficient $(>7)$ we used Student's t test for comparing two groups. Comparison of 4 groups were done with 2-way ANOVA and time courses were analyzed with 2-way repeated measures ANOVA, as indicated. Post hoc multiple comparisons after ANOVA were done with Holm-Sidak's test. All tests were used as two-tailed. Statistical analyses were done with GraphPad Prism 6. Estimation statistics was done as recommended ${ }^{46}$ using the https://www. estimationstats.com/ website ${ }^{47}$. Statistical analyses are reported in Supplementary Tables 1-5.

Received: 2 May 2021; Accepted: 27 July 2021

Published online: 11 August 2021 


\section{References}

1. Walkiewicz, K. W., Girault, J. A. \& Arold, S. T. How to awaken your nanomachines: Site-specific activation of focal adhesion kinases through ligand interactions. Prog. Biophys. Mol. Biol. 119, 60-71 (2015).

2. Bartos, J. A. et al. Postsynaptic clustering and activation of Pyk2 by PSD-95. J. Neurosci. 30, 449-463 (2010).

3. Kohno, T., Matsuda, E., Sasaki, H. \& Sasaki, T. Protein-tyrosine kinase CAKbeta/PYK2 is activated by binding Ca2+/calmodulin to FERM F2 alpha2 helix and thus forming its dimer. Biochem. J. 410, 513-523 (2008).

4. Lev, S. et al. Protein tyrosine kinase PYK2 involved in $\mathrm{Ca}(2+)$-induced regulation of ion channel and MAP kinase functions. Nature 376, 737-745 (1995).

5. Zwick, E., Wallasch, C., Daub, H. \& Ullrich, A. Distinct calcium-dependent pathways of epidermal growth factor receptor transactivation and PYK2 tyrosine phosphorylation in PC12 cells. J. Biol. Chem. 274, 20989-20996 (1999).

6. Faure, C. et al. Calcineurin is essential for depolarization-induced nuclear translocation and tyrosine phosphorylation of PYK2 in neurons. J. Cell Sci. 120, 3034-3044 (2007).

7. Menegon, A. et al. FAK+ and PYK2/CAKbeta, two related tyrosine kinases highly expressed in the central nervous system: Similarities and differences in the expression pattern. Eur. J. Neurosci. 11, 3777-3788 (1999).

8. Siciliano, J. C., Gelman, M. \& Girault, J. A. Depolarization and neurotransmitters increase neuronal protein tyrosine phosphorylation. J. Neurochem. 62, 950-959 (1994).

9. Siciliano, J. C., Toutant, M., Derkinderen, P., Sasaki, T. \& Girault, J. A. Differential regulation of proline-rich tyrosine kinase $2 /$ cell adhesion kinase beta (PYK2/CAKbeta) and pp125(FAK) by glutamate and depolarization in rat hippocampus. J. Biol. Chem. 271, 28942-28946 (1996).

10. Huang, Y. et al. CAKbeta/Pyk2 kinase is a signaling link for induction of long-term potentiation in CA1 hippocampus. Neuron 29, 485-496 (2001).

11. Avraham, H., Park, S. Y., Schinkmann, K. \& Avraham, S. RAFTK/Pyk2-mediated cellular signalling. Cell Signal 12, 123-133 (2000).

12. Li, X., Dy, R. C., Cance, W. G., Graves, L. M. \& Earp, H. S. Interactions between two cytoskeleton-associated tyrosine kinases: Calcium-dependent tyrosine kinase and focal adhesion tyrosine kinase. J. Biol. Chem. 274, 8917-8924 (1999).

13. Salter, M. W. \& Kalia, L. V. Src kinases: A hub for NMDA receptor regulation. Nat. Rev. Neurosci. 5, 317-328 (2004).

14. Seabold, G. K., Burette, A., Lim, I. A., Weinberg, R. J. \& Hell, J. W. Interaction of the tyrosine kinase Pyk2 with the N-methylD-aspartate receptor complex via the Src homology 3 domains of PSD-95 and SAP102. J. Biol. Chem. 278, 15040-15048 (2003).

15. Kim, E. \& Sheng, M. PDZ domain proteins of synapses. Nat. Rev. Neurosci. 5, 771-781 (2004).

16. Zhao, C. et al. The upregulation of NR2A-containing N-methyl-d-aspartate receptor function by tyrosine phosphorylation of postsynaptic density 95 via facilitating Src/proline-rich tyrosine kinase 2 activation. Mol. Neurobiol. 51, 500-511 (2015).

17. O’Dell, T. J., Kandel, E. R. \& Grant, S. G. Long-term potentiation in the hippocampus is blocked by tyrosine kinase inhibitors. Nature 353, 558-560 (1991).

18. Heidinger, V. et al. Metabotropic glutamate receptor 1-induced upregulation of NMDA receptor current: Mediation through the Pyk2/Src-family kinase pathway in cortical neurons. J. Neurosci. 22, 5452-5461 (2002).

19. Afonso, P., et al. BDNF increases synaptic NMDA receptor abundance by enhancing the local translation of Pyk 2 in cultured hippocampal neurons. Sci. Signal 12, eaav3577 (2019).

20. Hsin, H., Kim, M. J., Wang, C. F. \& Sheng, M. Proline-rich tyrosine kinase 2 regulates hippocampal long-term depression. J. Neurosci. 30, 11983-11993 (2010).

21. Giralt, A. et al. Pyk2 modulates hippocampal excitatory synapses and contributes to cognitive deficits in a Huntington's disease model. Nat. Commun. 8, 15592 (2017).

22. Salazar, S. V. et al. Alzheimer's disease risk factor Pyk2 mediates amyloid-beta-induced synaptic dysfunction and loss. J. Neurosci. 39, 758-772 (2019).

23. Aggleton, J. P. \& Nelson, A. J. D. Distributed interactive brain circuits for object-in-place memory: A place for time?. Brain Neurosci. Adv. 4, $2398212820933471(2020)$.

24. Assini, F. L., Duzzioni, M. \& Takahashi, R. N. Object location memory in mice: Pharmacological validation and further evidence of hippocampal CA1 participation. Behav. Brain Res. 204, 206-211 (2009).

25. Cunningham, J. I., Raudensky, J., Tonkiss, J. \& Yamamoto, B. K. MDMA pretreatment leads to mild chronic unpredictable stressinduced impairments in spatial learning. Behav. Neurosci. 123, 1076-1084 (2009).

26. Giralt, A. et al. PTK2B/Pyk2 overexpression improves a mouse model of Alzheimer's disease. Exp. Neurol. 307, 62-73 (2018).

27. Hammond, S. L., Leek, A. N., Richman, E. H. \& Tjalkens, R. B. Cellular selectivity of AAV serotypes for gene delivery in neurons and astrocytes by neonatal intracerebroventricular injection. PLoS ONE 12, e0188830 (2017).

28. Tsien, J. Z. et al. Subregion- and cell type-restricted gene knockout in mouse brain. Cell 87, 1317-1326 (1996).

29. Kugler, S., Lingor, P., Scholl, U., Zolotukhin, S. \& Bahr, M. Differential transgene expression in brain cells in vivo and in vitro from AAV-2 vectors with small transcriptional control units. Virology 311, 89-95 (2003).

30. Nicoll, R. A. A brief history of long-term potentiation. Neuron 93, 281-290 (2017).

31. Gerlai, R. Behavioral tests of hippocampal function: Simple paradigms complex problems. Behav. Brain Res. 125, 269-277 (2001).

32. Vorhees, C. V. \& Williams, M. T. Assessing spatial learning and memory in rodents. ILAR J. 55, 310-332 (2014).

33. Harrison, F. E., Hosseini, A. H. \& McDonald, M. P. Endogenous anxiety and stress responses in water maze and Barnes maze spatial memory tasks. Behav. Brain Res. 198, 247-251 (2009).

34. Dere, E., Huston, J. P. \& De Souza Silva, M. A. The pharmacology, neuroanatomy and neurogenetics of one-trial object recognition in rodents. Neurosci. Biobehav. Rev. 31, 673-704 (2007).

35. Huang, C. C. \& Hsu, K. S. Protein tyrosine kinase is required for the induction of long-term potentiation in the rat hippocampus. J. Physiol. 520(Pt 3), 783-796 (1999).

36. Grant, S. G. et al. Impaired long-term potentiation, spatial learning, and hippocampal development in fyn mutant mice. Science 258, 1903-1910 (1992).

37. Larson, J. \& Munkacsy, E. Theta-burst LTP. Brain Res. 1621, 38-50 (2015).

38. Abel, T. et al. Genetic demonstration of a role for PKA in the late phase of LTP and in hippocampus-based long-term memory. Cell 88, 615-626 (1997).

39. Nguyen, P. V. \& Kandel, E. R. Brief theta-burst stimulation induces a transcription-dependent late phase of LTP requiring cAMP in area CA1 of the mouse hippocampus. Learn Mem. 4, 230-243 (1997).

40. Woo, N. H., Duffy, S. N., Abel, T. \& Nguyen, P. V. Temporal spacing of synaptic stimulation critically modulates the dependence of LTP on cyclic AMP-dependent protein kinase. Hippocampus 13, 293-300 (2003).

41. Lau, C. G. \& Zukin, R. S. NMDA receptor trafficking in synaptic plasticity and neuropsychiatric disorders. Nat. Rev. Neurosci. 8, 413-426 (2007).

42. Gallistel, C. R. \& Balsam, P. D. Time to rethink the neural mechanisms of learning and memory. Neurobiol. Learn Mem. 108, 136-144 (2014)

43. Titley, H. K., Brunel, N. \& Hansel, C. Toward a neurocentric view of learning. Neuron 95, 19-32 (2017).

44. Etienne, F., et al. Two-photon imaging of microglial processes' attraction toward ATP or serotonin in acute brain slices. J. Vis. Exp. 143, e58788 (2019). 
45. Ting, J. T., Daigle, T. L., Chen, Q. \& Feng, G. Acute brain slice methods for adult and aging animals: Application of targeted patch clamp analysis and optogenetics. Methods Mol. Biol. 1183, 221-242 (2014).

46. Calin-Jageman, R.J. \& Cumming, G. Estimation for better inference in neuroscience. eNeuro 6 0205-19 (2019).

47. Ho, J., Tumkaya, T., Aryal, S., Choi, H. \& Claridge-Chang, A. Moving beyond P values: Data analysis with estimation graphics. Nat. Methods 16, 565-566 (2019).

\section{Acknowledgements}

The authors thank Nicolas Gervasi and Giulia Albertini for their help in the initial set up of electrophysiological and behavioral test respectively.

\section{Author contributions}

J.A.G., V.M., O.M. designed research; V.M., O.M., B.P. performed experiments; V.M., O.M., B.P., J.A.G. analyzed data; V.M., O.M., J.A.G. wrote the paper.

\section{Funding}

BdP was supported by the Fondation pour la recherche médicale (FRM, FDT201805005390). The work was supported by grants to JAG from Agence Nationale de la Recherche (ANR-15-NEUC-0002-02 and ANR19-CE16-0020), FRM (EQU201903007844) and Inserm-Transfert COPOC. Omar Al-Massadi is currently funded by a research contract "Miguel Servet" (CP20/00146) from the Instituto de Salud Carlos III (ISCIII), co-financed by the European Social Fund (FSE). CIBERobn is an initiative of the ISCIII of Spain, which is supported by FEDER funds.

\section{Competing interests}

The authors declare no competing interests.

\section{Additional information}

Supplementary Information The online version contains supplementary material available at https://doi.org/ 10.1038/s41598-021-95813-x.

Correspondence and requests for materials should be addressed to J.-A.G.

Reprints and permissions information is available at www.nature.com/reprints.

Publisher's note Springer Nature remains neutral with regard to jurisdictional claims in published maps and institutional affiliations.

(c) (i) Open Access This article is licensed under a Creative Commons Attribution 4.0 International License, which permits use, sharing, adaptation, distribution and reproduction in any medium or format, as long as you give appropriate credit to the original author(s) and the source, provide a link to the Creative Commons licence, and indicate if changes were made. The images or other third party material in this article are included in the article's Creative Commons licence, unless indicated otherwise in a credit line to the material. If material is not included in the article's Creative Commons licence and your intended use is not permitted by statutory regulation or exceeds the permitted use, you will need to obtain permission directly from the copyright holder. To view a copy of this licence, visit http://creativecommons.org/licenses/by/4.0/.

(C) The Author(s) 2021 